\title{
HOMOGENEOUS POLYNOMIALS WITH A MULTIPLICATION THEOREM*
}

\author{
BY \\ O. C. HAZLETT
}

1. Relation to the literature. If $f(x)$ is any homogeneous polynomial in the $n$ variables, $x_{1}, \cdots, x_{n}$, such that

$$
f(x) f(\xi)=f(X)
$$

where the $X$ 's are bilinear functions of the $x$ 's and $\xi$ 's,

$$
X_{k}=\sum_{i j} \gamma_{i j k} x_{i} \xi_{i} \quad(k=1, \cdots, n),
$$

then $f(x)$ is said to admit the composition (2).

One of the simplest such functions is $f(x)=x_{1}^{2}+x_{2}^{2}$ for which

$$
X_{1}=x_{1} \xi_{1} \pm x_{2} \xi_{2}, \quad X_{2}=x_{2} \xi_{1} \mp x_{1} \xi_{2} .
$$

This was known to Diophantus. $\dagger$ Euler and Degen noticed that, similarly, a sum of $n$ squares admits composition when $n=4$ or 8 , and Hurwitz proved that such is true only when $n=1,2,4,8$. Two other well known examples are the multiplication theorem for determinants whose $n^{2}$ elements are independent variables and the norms of algebraic numbers. Moreover the composition of forms occurs in number theory.

Usually in number theory we are concerned with the composition of forms in a more general sense, viz,

$$
f^{\prime}(x) f^{\prime \prime}(\xi)=F(X)
$$

where $f^{1}, f^{11}$ and $F$ are polynomials in the same number of variables and of the same degree, but not necessarily with the same coefficients. Dickson§ proved that the determination of three polynomials satisfying (4) can be reduced to the determination of one polynomial satisfying (1). Hence we can and shall restrict our attention to compositions (1).

\footnotetext{
* Presented to the Society, December 29, 1926; received by the editors in July, 1928.

$\dagger$ For the history and bibliography of the subject of composition of forms, see Dickson's $B$ istory of the Theory of Numbers, vol. III, especially Chapters III, IV.

$\ddagger$ For a simple exposition and an account of the history of this problem, see Dickson, Annals of Mathematics, (2), vol. 20 (1919), pp. 155-171, 297.

8 Comptes Rendus, Paris, vol. 172 (1921), pp. 636-640.
} 
In another illuminating article, ${ }^{*}$ Dickson pointed out that the determination of all polynomials $f(x)$ which satisfy (1) where the $X_{k}$ are defined in terms of the $x$ and $\xi$ by (2) is equivalent to the determination of all polynomials $f(x)$ which satisfy (1) where $x \xi=X$ in the linear algebra $E$ of order $n$ whose multiplication table is

$$
e_{i} e_{j} \equiv \sum_{k} \gamma_{i j k} e_{k} \quad(i, j=1, \cdots, n) .
$$

The determinant of the $\xi_{j}$ in (2) is

$$
\Delta(x)=\left|\sum_{i} \gamma_{i j k} x_{i}\right|,
$$

the first (or right-hand) determinant of the number $x=\sum x_{i} e_{i}$ in the algebra $E$. When $f(x)=x_{1}{ }^{2}+x_{2}{ }^{2}$ and the composition is given by (3), then the algebra (5) is $e_{1} e_{1}=e_{1}, e_{1} e_{2}=\mp e_{2}, e_{2} e_{1}=e_{2}, e_{2} e_{2}= \pm e_{1}$. When $f(x)=x_{1}^{2}+x_{2}^{2}+x_{3}^{2}+x_{4}^{2}$ and the composition is $X_{1}=\xi_{1} x_{1}-\xi_{2} x_{2}-\xi_{3} x_{3}-\xi_{4} x_{4}, X_{2}=\xi_{2} x_{1}+\xi_{1} x_{2}+\xi_{4} x_{3}-\xi_{3} x_{4}$, $X_{3}=\xi_{3} x_{1}-\xi_{4} x_{2}+\xi_{1} x_{3}+\xi_{2} x_{4}, X_{4}=\xi_{4} x_{1}+\xi_{3} x_{2}-\xi_{2} x_{3}+\xi_{1} x_{4}$, then the corresponding algebra is real quaternions with $e_{1} \equiv 1, e_{2}=i, e_{3}=j, e_{4}=k$; whereas $f(x)$ $=\sum x_{i}^{2}(i=1, \cdots, 8)$ admits composition under Cayley's algebra of eight units with the multiplication table

$$
\begin{aligned}
e_{i}{ }^{2} & =-1, \quad e_{i} e_{j}=-e_{i} e_{i} \quad(i, j=2, \cdots, 8 ; i \neq j) ; \\
e_{2} e_{3} & =e_{4}, \quad e_{2} e_{5}=e_{6}, \quad e_{2} e_{7}=e_{8}, \quad e_{3} e_{6}=e_{8}, \quad e_{3} e_{5}=-e_{7}, \\
e_{4} e_{5} & =e_{8}, \quad e_{4} e_{6}=e_{7} .
\end{aligned}
$$

If we take the lower sign in the multiplication table of the binary algebra given above, we see that the sum of two squares admits composition under an algebra which is both associative and commutative, but in order to get an algebra under which the sum of four squares admits composition, we have to discard the commutative law, and finally, to get an algebra under which the sum of eight squares admits composition, we have to discard the associative law, also. This makes it not at all surprising that there is no linear algebra under which the sum of 16 or more squares admits composition.

Dickson proved the following:

THEOREM A. If $f(x)$ is a homogeneous polynomial of degree $d$ which admits a composition and is not expressible in fewer than $n$ variables, we may assume that the coefficient of $x_{1}{ }^{d}$ is unity after applying a transformation cogredient in the $x$ 's, $\xi$ 's and X's. Then by applying a transformation on the X's which leaves

*Homogeneous polynomials with a multiplication theorem, Comptes Rendus du Congrès International des Mathématiciens, Strasbourg, 1921. 
$f(x)$ unaltered and a transformation on the $\xi$ 's which leaves $f(\xi)$ unaltered, we obtain for $f(x)$ a new composition $x \xi=X$ in a linear algebra having a principal unit $e_{1}$ for which $e_{1} e_{j}=e_{j}=e_{j} e_{1}(j=1, \cdots, n)$.

Such a composition is said to be normalized.

THEOREM B. If $f(x)$ admits normalized composition, any factor $E(x)$ of $f(x)$ admits the composition if it has unity as the coefficient of the term involving only $x_{1}$.

THEOREM C. If a polynomial admits normalized composition, each of its irreducible factors (with the convention of Theorem B) admits the composition and divides both $\Delta(x)$ and $\Delta^{\prime}(x)$.

After finding all forms in 2, 3 and 4 variables which admit composition in the field of complex numbers, Dickson remarks that he has not yet succeeded in deciding whether or not an irreducible form of degree 5 in 5 variables admits composition.

2. General case. Let $f(x)$ be a homogeneous polynomial in $n$ variables of degree $d$ which admits the composition (2) and which is not expressible as a polynomial in less than $n$ variables. If we give to the $n x$ 's any set of values such that $f(x)=c \neq 0$, then $f\left(c^{1 / d} \xi\right)=f(X)$. Since $c \neq 0$, then $c^{1 / d} \xi_{i}(i=1, \cdots$, $n$ ) are $n$ linearly independent variables. Hence, since $f$ can not be expressed as a function of less than $n$ variables (by a non-singular linear transformation), the $n X$ 's must be linearly independent and therefore $\Delta(X) \neq 0$. But $\Delta(X)=\Delta(x) \Delta(\xi)$ and therefore $\Delta(x) \neq 0$.

Next, give to the $x$ 's such a constant set of values that $f(x)=0$ and let the $\xi$ 's be independent variables. Then $\Delta(x)=0$. For if $\Delta(x) \neq 0$, we should have $f(X) \equiv f(x) f(\xi) \equiv 0$ when the $X$ 's are $n$ linearly independent functions of the $\xi$ 's and therefore are $n$ linearly independent variables. Since this is a contradiction (except in the trivial case when $f(x)$ is originally assumed to be identically zero), our assumption that $\Delta(x) \neq 0$ is false.

Combining the two results, we see that $\Delta(x)=0$ is equivalent to $f(x) \equiv 0$. Similarly, $\Delta^{\prime}(x)=0$ is equivalent to $f(x)=0$ and we have proved

THEOREM 1. If $f(x)$ is a polynomial in $n$ variables which admits composition and which can not be expressed as a polynomial in less than $n$ variables, then $f(x)$ has precisely the same irreducible factors as $\Delta(x)$ and $\Delta^{\prime}(x)$, the first and second determinants of the linear algebra defined by the composition.

When $n<5$, this was proved by Dickson.* Moreover, it is readily verified that this is true for the case when $f(x)=\sum x_{i}{ }^{2}(i=1, \cdots, 8)$ and $E$ is Cayley's

* His Strasbourg paper mentioned above, Theorem 5 . 
algebra of order eight whose multiplication table is given by (6).

COROLLARY. The degree of an irreducible factor $f(x)$ of a function admitting normalized composition is a factor of the minimum number of variables necessary to express $f(x)$.

For let $E$ be the algebra defined by the composition and let the variables necessary to express $f$ be denoted by $x_{1}, \cdots, x_{n}$. Then $f$ admits composition under $E$ and under the algebra obtained from $E$ by deleting all numbers except those of the form $x_{1} e_{1}+\cdots+x_{n} e_{n}$, the equations defining this latter composition being (2). But the order of this algebra is $n$ and thus $\Delta$ is of degree $n$; and, by Theorem $1, \Delta$ is a power of $f(x)$. Thus the corollary is proved.

3. Powers of an irreducible polynomial. Combining Theorem 1 with Theorem B, we have

THEOREM 2. Let $f(x)$ be a homogeneous polynomial in $n$ variables admitting normalized composition (2) under the linear algebra $E$ and such that no one of its factors can be expressed as a polynomial in less than $n$ variables. Then $f(x)$ is a power of $\Delta(x)$, and $\Delta(x)$ is either irreducible or is a power of an irreducible function. Similarly for $\Delta^{\prime}(x)$.

We also readily prove Theorem 6 of Dickson's paper for general $n$ (which he had proved only for $n<5$ ), namely

THEOREM 3. If a form $f$ in $n$ variables admits normalized composition and has an irreducible factor $I$ which is not expressible in less than $n$ variables, then $f$ is a power of $I$.

For, since $I$ admits the composition and is not expressible as a polynomial in less than $n$ variables, then Theorem 1 applies and thus $\Delta$ is a power of $I$. But $I$ is irreducible and $f$ has no factors other than those of $I$. Hence $f$ is a power of $I$.

THEOREM 4. If $f(x)$ is a polynomial in $n$ variables $(n>1)$ which admits composition and which can not be expressed as a polynomial in a smaller number of variables, then $f(x)$ is not irreducible of degree $n$ in the field of complex numbers. On the other hand, if the field of definition is any field $F$, then the only such functions are the norms of numbers which are algebraic with respect to $F$.

Without loss of generality, we shall prove this when the algebra is normalized. Since $f(x)$ is of degree $n$ and is irreducible, then by Theorem 2, $\Delta(x)$ must be irreducible and identical with $f(x)$. Now the (first) characteristic equation of the general number $x$ of the algebra is $\delta(x) \equiv \Delta(x-\omega \epsilon)=0$ where 
$\epsilon$ is the principal unit or modulus and thus $\delta(x)$ is irreducible for the general number. Hence the entire algebra is generated by one number of the algebra and hence must be a field which is algebraic with respect to $F$ of order $n$. But when $F$ is the field of complex numbers, this is possible only when $n=1$.

THEOREM 5. If $f(x)$ is a form in $n$ variables $x_{1}, \cdots, x_{n}$ which admits composition and which vanishes only when $x_{1}=x_{2}=\cdots=x_{n}=0$, then the algebra defined by the composition is a division algebra; and conversely.

Since $f(x)$ vanishes only when $x_{1}=x_{2}=\cdots=x_{n}=0$, it follows that no factor of $f(x)$ is expressible in less than $n$ variables. For, if some factor were expressible in $n_{1}$ variables $\left(n_{1}<n\right)$, we may without loss of generality assume these variables to be $x_{1}, \cdots, x_{n_{1}}$. Then $f(x)$ would vanish when $x_{1}=x_{2}$ $=\cdots=x_{n_{1}}=0$ and $x_{i}\left(i>n_{1}\right)$ is arbitrary, which is a contradiction. Hence $f(x)$ is a power of $\Delta(x)$ and thus $\Delta(x)$ vanishes if and only if $x_{1}=x_{2}=\cdots=x_{n}$ $=0$. Since, in any linear algebra, $\Delta(x)=0$ is a necessary and sufficient condition that $x$ be a divisor of zero, it follows that the algebra defined by the composition has no divisors of zero and hence is a division algebra. This proves the first half of the theorem and the converse is proved by reversing the argument.

THEOREM 6. If $f(x)$ is a power of an irreducible form in $n$ variables which is not expressible in less than $n$ variables, then the algebra defined by the composition is irreducible.

For since $f(x)$ is a power of an irreducible polynomial in not less than $n$ variables, so also is $\Delta(x)$ by Theorem 2 . If $E$ were reducible, then its basal numbers could be so chosen that they could be divided into two sets, the $e$ 's and $\vec{e}$ 's, such that the product of two basal numbers from the same set is linearly dependent on the numbers of that set but such that the product of any two basal numbers from different sets is zero. Hence the first determinant, $\Delta(x)$, for the general number of the algebra is of the form $\Delta_{1}(x) \Delta_{2}(x)$, where $\Delta_{1}(x)$ is the first determinant for the general number of the subalgebra determined by the $e$ 's and $\Delta_{2}(x)$ is the first determinant for the general number of the subalgebra determined by the $\bar{e}$ 's. Hence, if $E$ were reducible, we should have a contradiction.

4. Two or more irreducible factors. Next, we shall consider the case when $f(x)$ is reducible but is not a power of an irreducible function. By Theorem 1, we may assume without loss of generality that $f(x)=\Delta(x)$. Let $f(x)=\Pi_{i}\left(f_{i}(x)\right)^{p 1}$, where each $f_{i}$ is an irreducible polynomial and where $f_{1}, f_{2}, \cdots$ are distinct. By Theorem 3 , every one of the polynomials $f_{1}, f_{2}, \cdots$ can be expressed as a polynomial in less than $n$ variables. Moreover, since 
any factor of $f(x)$ (with the convention used in Theorem B) admits the composition, it follows from Theorem 1 that any factor of $f(x)$ can be expressed as a polynomial in less than $n$ variables unless it contains each of the factors $f_{i}(x)$ with non-zero exponent.

As indicated above, let the composition be normalized with $e_{1}$ as the modulus of the algebra. Then, since $f_{1}(x)$ can be expressed as a polynomial in less than $n$ variables, make a linear transformation on the variables $x_{i}$ so that $f_{1}(x)$ is a polynomial in $x_{1}, \cdots, x_{n_{1}}$, in which $n_{1}$ is as small as possible. When we make the induced transformation on the basal numbers of the algebra, the modulus of the algebra is not in general $e_{1}$ and so the composition is not normalized, strictly speaking, although the composition still posseses the essential feature of normalized composition, namely, the algebra has a modulus or principal unit. Moreover, although the composition is no longer normalized, yet suitably chosen irreducible factors admit the composition, namely, the transforms of the original $f_{i}(x)$. Since the product of the rest of the $f_{i}(x)$ is expressible in less than $n$ variables and since the totality of variables necessary in all the $f_{i}$ taken together is $n$, then we may so choose the variables necessary to express $\phi_{1}=\Pi f_{i}(i>1)$ as $x_{n_{1+1}}, \cdots, x_{n}$ with, possibly, one or more chosen out of the set $x_{1}, \cdots, x_{n_{1}}$ and these latter we may, without loss of generality, assume to be $x_{i_{1}+1}, \cdots, x_{n_{1}}\left(i_{1}>0\right)$.

First we prove that $f(x)$ is a product of polynomials in non-overlapping sets of variables. To prove this statement, we prove that the coefficient of any term in $f_{1}$ which contains the variables $x_{k_{1}}\left(k=i_{1}+1, \cdots, n_{1}\right)$ is necessarily zero, and proceed by induction.

In $f(x)$, set equal to zero every $x_{k}$ where $k<i_{1}$. Then, if $f_{1}$ actually contain terms in the $x_{k}$ alone where $k>i_{1}$, the result of making this substitution in $f$ is a polynomial $g$ in $n-i_{1}$ variables which is not identically zero and which admits the composition $g(x) g(\xi)=g(X)$ under the algebra $B_{1}$ obtained from $E$ by deleting the numbers $x_{1} e_{1}+\cdots+x_{i_{1}} e_{i_{1}}{ }^{*}$ Since $g(x)$ and $\phi_{1}(x)$ admit composition under the same algebra $B_{1}$ and neither can be expressed in a smaller number of variables, then (by Theorem 1) $g(x)$ has the same irreducible factors as $\phi_{1}(x)$ and thus $\phi_{1}(x)$ has at least one factor which contains no variables from the set $x_{j}\left(j=n_{1}, \cdots, n\right)$. By regrouping the factors of $f(x)$, we can write $f(x)$ in the form $g_{1}(x) g_{2}(x)$ where $g_{1}(x)$ is a polynomial in the first $n_{1}$ variables and where $g_{2}(x)$ is a polynomial containing no factor in the first $n_{1}$ variables alone. If we now apply the preceding argument, we have a contradiction unless $g_{2}(x)$ were independent of the first $n_{1}$ variables. Hence

* Note that this is not the same as the statement that the totality of numbers of the form $M x_{j} e_{j}\left(j \leqq i_{1}\right)$ form an algebra. 
if $f_{1}(x)$ actually contain a term in the $x_{j}\left(j>i_{1}\right)$ alone, then $f$ can be written in the form $g_{1} g_{2}$ where $g_{1}$ is a polynomial in the first $n_{1}$ variables and $g_{2}$ is a polynomial in the rest of the variables - two independent sets of variables. Moreover, since $g_{1}$ is a polynomial in $n_{1}$ variables that admits composition under the algebra obtained from $E$ by deleting all except the first $n_{1}$ basal numbers, it follows (by Theorem 3 and the understanding about the variables) that $g_{1}$ is a power of $f_{1}$. This result is proved under the hypothesis that $f_{1}$ actually contains a term in the $x_{j}$ alone $\left(j>i_{1}\right)$. Since this hypothesis leads to a contradiction, it follows that $f_{1}$ contains no term in these $x_{j}$ alone. Here it is understood that $f_{1}$ can not be expressed in less than $n_{1}$ variables and that these $x_{i}$ are the variables that actually appear in $f_{1}$ and also in the other $f_{k}$.

From this it follows that $f_{1}$ contains no term whatsoever in these $x_{j}\left(j>i_{1}\right)$, i.e., it can be expressed in less than $n_{1}$ variables. For if $f_{1}$ could not be expressed in less than $n_{1}$ variables, then we should be making an allowable transformation, that is, one that still expresses $f_{1}$ in the least number of variables, when we replace every $x_{k}$ by $x_{k}+a_{k} x_{n_{1}}\left(k<n_{1}\right)$. The coefficient of the term in $x_{n_{1}}$ alone in the transformed $f_{1}$ would be $f_{1}\left(a_{1}, a_{2}, \cdots, a_{n_{1}-1}, 1\right)$. But, since $f_{1}$ is not identically zero, there is a set of numbers in the given field $F$ such that this coefficient is not zero. Since this is a contradiction of the preceding paragraph, it follows that $f_{1}$ can be expressed in less than $n_{1}$ variables.

But this last conclusion is inconsistent with the significance of the notation for the variables, and thus the two sets of variables necessary to express $f_{1}$ and $\phi_{1}$ are entirely independent of each other. Now $\phi_{1}$ is a polynomial in $n-n_{1}$ variables which admits composition under $E$ and hence under the algebra obtained from $E$ by deleting the first $n_{1}$ basal numbers. Hence, by induction, it follows that $f$ is a product of powers of irreducible polynomials in non-overlapping sets of variables.

This means that the composition (1) depends only on those $\gamma_{i j k}$ where $i, j, k$ are all chosen from the set $1, \cdots, m_{1}=n_{1}$ or all chosen from the second set $m_{1}+1, \cdots, m_{2}$, or all chosen from one of the sets $m_{p-1}+1, \cdots$, $m_{p}$ where $m_{p}=\sum n_{i}(i=1, \cdots, p)$. The others may not be zero, conceivably; but they do not actually appear in $f(X)$ after like terms have been combined. For, since $f_{1}(x) f_{1}(\xi)=f_{1}(X)$, where only the first set of variables appears in $f_{1}$, it follows that although $X_{k}=\sum \gamma_{i j k} x_{i} \xi_{j}\left(k=1, \cdots, n_{1}\right)$, yet the terms in the variables $\xi$ and $x$ from sets other than the first must cancel in $f_{1}(X)$ and thus may be ignored so far as $f_{1}$ is concerned. Since these same variables do not appear in any other $f_{i}$, they do not affect $f$ or the validity of the composition. Hence we may assume to be zero every $\gamma_{i j k}$ in which all three subscripts 
are not in the same set, and we have

THEOREM 7. Let $f(x)$ be a homogeneous polynomial in $n$ variables which admits composition and which can not be expressed in less than $n$ variables. Then, if $f(x)$ is not the power of an irreducible polynomial, it is equivalent (under a linear transformation on the variables) to a product of powers of distinct irreducible forms, $f_{i}(x)$, where (1) $f_{i}$ is a form in $n_{i}$ variables which can not be expressed as a form in a smaller number of variables; (2) the various sets of variables are non-overlapping; (3) $\sum n_{i}=n$; (4) if $E$ is any algebra defined by the composition, then $f(x)$ admits composition under the algebra obtained from $E$ by replacing by zero every $\gamma_{i j k}$ where $i, j, k$ are not all from the same set (for details, see the proof of the theorem).

5. Applications. In view of the last theorem, we see that, in order to determine all homogeneous polynomials in $n$ variables which admit a composition and which are not expressible in less than $n$ variables, we first determine all such irreducible polynomials of the type just described together with products of powers of homogeneous polynomials $f_{i}$ in non-overlapping sets of variables such that each $f_{i}$ admits composition in the variables appearing in $f_{i}$, in the manner described in detail in Theorem 7.

There are two possibilities: (a) $\Delta(x)$ is expressible as a polynomial in less than $n$ variables; (b) $\Delta(x)$ is not so expressible. Since, in this paper, we are interested in finding all polynomials, $f(x)$, which admit composition, but are not concerned with the problem of determining all compositions (2) under which a given $f(x)$ admits composition, we can ignore the first possibility since a polynomial which can be expressed as a polynomial in a smaller number of variables can and will be considered as a polynomial in the smallest possible number of variables. For the same reason, if $f(x)$ is reducible, we shall consider merely the most convenient algebra defined by the composition, namely the reducible algebra determined in the proof of Theorem 7 .

First, let $f(x)$ be a form of degree $n$ in $n$ variables with coefficients in the field $F$ and let it be irreducible there. Then, by Theorem $4, f(x)$ must be the norm of the general number of a field which is algebraic with respect to $F$ of order $n$.

If $f(x)$ be of degree $n$ but reducible in $F$, there are two possibilities: $f(x)$ is a power of an irreducible polynomial in $F ;(2) f(x)$ is the product of powers of two or more distinct forms, $f_{1}(x), f_{2}(x)$, etc., each of which is irreducible in $F$ but which is expressible in a smaller number of variables than $n$. In case (2), by Theorem 7 , there is an algebra $E$ defined by the composition on all $n$ variables which is reducible and such that it is the direct sum of two or more algebras such that the assertion that $f(x)$ admits 
composition under this algebra $E$ is equivalent to the assertion that $f_{i}(x)$ admits composition under the algebra $E_{\boldsymbol{i}}$ for every $i$. Accordingly, to determine all such polynmials $f(x)$, we determine all such polynomials $f_{1}(x), f_{2}(x), \cdots, f_{k}(x)$ such that (i) $f_{1}(x)$ is a polynomial in $n_{1}$ variables, but not in a smaller number of variables, with similar conventions about $f_{2}(x)$, etc.; (ii) if the variables necessary for $f_{1}(x)$ be denoted by $x$ with subscripts ranging from 1 to $n_{1}$ inclusive, and if the variables necessary for $f_{2}(x)$ be denoted by $x$ with subscripts ranging from $n_{1}+1$ to $n_{1}+n_{2}$ inclusive with similar notations for the variables necessary for the remaining $f(x)$, then $n_{1}+n_{2}+\cdots+n_{k}=n$.

Third, let $f(x)$ be reducible, but the power of an irreducible polynomial, $\phi(x)$, of degree $d$. Then $d$ is a factor of $n$, the number of variables needed. If the algebra defined by the composition is associative, then $E$ must be semisimple (that is, containing no invariant nilpotent sub-algebra). For if $E$ is not semi-simple, then $E=S+N$ where $S$ is a semi-simple algebra and $N$ is a maximal invariant nilpotent sub-algebra, and it is well known that the characteristic determinant $\Delta(x)$ formed for the general number $x$ of the algebra $E$ is the product of $\Delta_{s}(x)$, the characteristic determinant of the component of $x$ in $S$, by a determinant $D_{n}(x)$. Now it is readily proved (for any field $F$ ), that the irreducible factors of $D_{n}(x)$ are the same as the irreducible factors of $\Delta_{s}(x)$. Hence, when the algebra may be taken as associative, $\Delta(x)$ is expressible as a polynomial in less than $n$ variables. If, however, the algebra may not be taken as associative, not sufficient is at present known about non-associative algebras to enable us to decide whether a similar result is true here, also.

Thus we see that if $f(x)$ is a form in $n$ variables which admits composition, then we have the following results for small values of $n$ :

THEOREM 8. When $n=2, f(x)$ is a power of an irreducible form $\phi(x)$ of degree 2, where $\phi(x)$ is the norm of the general number of an algebraic field of order 2 with respect to $F$, or $f(x)$ has to be a product of powers of 2 linearly independent linear forms. When $F$ is $C$, the field of complex numbers, $f(x)$ has to be a product of powers of 2 linearly independent linear forms.

THEOREM 9. When $n=3, f(x)$ has to be a power of an irreducible form $\phi(x)$ of order 3 , where $\phi(x)$ is the norm of the general number of an algebraic field of order 3 with respect to $F$; or a product of powers of an irreducible form $\psi(x)$ of degree 2 in 2 variables, $x_{1}, x_{2}$, where $\psi(x)$ is the norm of the general number of $a$ field which is algebraic of order 2 with respect to $F$, and a linear form in a third variable, $x_{3}$; or it has to be a product of powers of 3 linearly independent linear forms in 3 variables. 
TheOREM 10. When $n=4, f(x)$ is one of the following:

(i) a power of an irreducible form $\phi(x)$ of degree 4 , where $\phi(x)$ is the norm of the general number of a field which is algebraic of order 4 with respect to $F$;

(ii) a power of an irreducible form in 4 variables of degree 2;

(iii) a product of powers of an irreducible cubic in 3 variables, $x_{1}, x_{2}, x_{3}$, and the linear form, $x_{4}$;

(iv) a product of powers of two irreducible quadratics in 2 variables, the first in $x_{1}, x_{2}$ and the second in $x_{3}, x_{4}$;

(v) a product of powers of an irreducible quadratic in 2 variables, $x_{1}, x_{2}$, and of two linear forms, $x_{3}, x_{4}$;

(vi) a product of powers of the 4 linear forms, $x_{1}, x_{2}, x_{3}, x_{4}$. Each form mentioned above which is irreducible of degree $k$ in $k$ variables is the norm of the general number of a field which is algebraic with respect to $F$ of order $k$.

When $F$ is the field of complex numbers, $f(x)$ has to be $a$ power of an irreducible quadratic in 4 variables or a product of powers of 4 linearly independent linear forms.

THEOREM 11. When $n=5, f(x)$ has to be one of the following:

(i) a power of an irreducible form $\phi(x)$ of degree 5, which is the norm of the general number of a field which is algebraic of order 5 with respect to $F$;

(ii) a product of powers of irreducible forms, $f_{i}(x)$, where each $f_{i}(x)$ is an irreducible form in less than 5 variables which admits composition, such that the variables actually necessary for any two or more $f_{i}(x)$ are linearly independent, and such that the total number of variables necessary is 5 .

In general, when $n$ is a prime, $f(x)$ has to be a power of an irreducible form $\phi(x)$ of degree $n$, which is the norm of the general number of a field which is algebraic of order $n$ with respect to $F$, or it has to be a product of powers of irreducible forms $f_{i}(x)$ in less than $n$ variables which admits composition, such that the variables necessary for any two or more $f_{i}(x)$ are linearly independent and such that the total number of variables necessary is $n$.

When $n$ is composite, $f(x)$ has to be of one of the two last mentioned types or it is a power of an irreducible form of degree $q$ in $n$ variables where $q$ is a divisor of $n$.

UNIVERSITY OF ILLINOIS, URBANA, II.L. 